\title{
Erratum
}

\section{Higher algebraic K-theory for actions of diagonalizable groups}

\author{
Gabriele Vezzosi ${ }^{1}$, Angelo Vistoli ${ }^{2}$ \\ 1 Dipartimento di Matematica Applicata "G. Sansone", Via S. Marta 3, 50139 Firenze, Italy \\ (e-mail: gabriele.vezzosi@unifi.it) \\ 2 Dipartimento di Matematica, Università di Bologna, 40126 Bologna, Italy \\ (e-mail: vistoli@dm.unibo.it)
}

Invent. math. 153, 1-44 (2003)

DOI: $10.1007 / \mathrm{s} 00222-002-0275-2$

Oblatum 18-VIII-2004 \& 18-XI-2004

Published online: 7 April 2005 - (C) Springer-Verlag 2005

Amnon Neeman has noticed a serious error in the proof of [Ve-Vi-2, Theorem 3.2]: the argument given does not yield a uniquely defined specialization map $\mathrm{Sp}_{Y}$, so that in particular compatibility with pullbacks does not hold. This is due to the elementary fact, overlooked in [Ve-Vi-2], that if one has a fiber sequence of spectra

$$
E \stackrel{f}{\longrightarrow} E^{\prime} \stackrel{g}{\longrightarrow} E^{\prime \prime},
$$

then a map $h: E^{\prime} \rightarrow E$ such that $h \circ f$ is homotopic to zero, does induce a map of spectra $p: E^{\prime \prime} \rightarrow E$, but this map is not unique, as it can be modified by using any map $E[1] \rightarrow E$ (by adding to any given $p$ the composite $E^{\prime \prime} \rightarrow E[1] \rightarrow E$ ). Of course if the nullhomotopy $h \circ f \sim 0$ is specified then this singles out a unique map $p: E^{\prime \prime} \rightarrow E$; but it is not clear to the authors how to choose a homotopy; thus, they are unable to define a specialization map in the generality claimed in the statement of [Ve-Vi-2, Theorem 3.2]. Despite their inability, the authors are firmly convinced that Theorem 3.2 holds, that is, that there exists a canonical specialization homomorphism, compatible with pullbacks.

Fortunately, it is still possible to define the specialization homomorphisms for higher K-theory in a generality that is sufficient for the rest of the paper: thus, all the results in Sects. 4, 5, 6 and 7, including the two 
main theorems, still hold unchanged. Also, Sect. 2 which is independent of Sect. 3 where specializations were defined, remains unchanged.

In what follows we will work in the same setup as in the paper, to which we refer for the unexplained notation. If $Y$ is a closed subscheme of a scheme $X$ over a fixed base $S$, we denote by $\mathrm{M}_{Y}^{0} X \rightarrow \mathbb{P}_{S}^{1}$ the deformation to the normal bundle, as in [Ful, Chapter 5], and in the paper. We denote by $\infty$ the closed subscheme of $\mathbb{P}_{S}^{1}$ that is the image of the section at infinity $S \rightarrow \mathbb{P}_{S}^{1}$; the inverse image of $\infty$ in $\mathrm{M}_{Y}^{0} X$ is the normal bundle $\mathrm{N}_{Y} X$.

Assume that $X$ is a regular noetherian algebraic space with the action of a diagonalizable group $G, Z$ a $G$-invariant regular Cartier divisor with trivial normal bundle, $i: Z \hookrightarrow X$ and $j: X \backslash Z \subseteq X$ the embeddings. The composition

$$
\mathrm{K}_{*}(Z, G) \stackrel{i_{*}}{\longrightarrow} \mathrm{K}_{*}(X, G) \stackrel{i^{*}}{\longrightarrow} \mathrm{K}_{*}(Z, G)
$$

is 0: if we assume that $j^{*}: \mathrm{K}_{*}(X, G) \rightarrow \mathrm{K}_{*}(X \backslash Z, G)$ is surjective, then we have an exact sequence

$$
0 \rightarrow \mathrm{K}_{*}(Z, G) \stackrel{i_{*}}{\longrightarrow} \mathrm{K}_{*}(X, G) \stackrel{j^{*}}{\longrightarrow} \mathrm{K}_{*}(X \backslash Z, G) \rightarrow 0
$$

hence the homomorphism $i^{*}: \mathrm{K}_{*}(X, G) \rightarrow \mathrm{K}_{*}(Z, G)$ factors through $\mathrm{K}_{*}(X \backslash Z, G)$, inducing a specialization ring homomorphism

$$
\mathrm{Sp}_{Z}^{X}: \mathrm{K}_{*}(X \backslash Z, G) \rightarrow \mathrm{K}_{*}(Z, G)
$$

If we restrict to $\mathrm{K}_{0}$, then surjectivity holds, and this is already in [SGA6, X-Appendice, 7.10].

Recall that $X_{s}$ is the regular subscheme of $X$ where the stabilizers have fixed dimension $s$, and that we have set $\mathrm{M}_{s} \stackrel{\text { def }}{=} \mathrm{M}_{X_{s}}^{0} X_{\leq s} \rightarrow \mathbb{P}^{1}$. Consider the closed embedding $\mathrm{N}_{s} \subseteq \mathrm{M}_{s}$, whose complement is $X_{\leq s} \times \mathbb{A}^{1}$. Looking at the composition

$$
X_{\leq s} \times \mathbb{A}^{1} \hookrightarrow \mathrm{M}_{s} \rightarrow X_{\leq s} \times \mathbb{P}^{1} \rightarrow X_{\leq s}
$$

we see that the pullback $\mathrm{K}_{*}\left(\mathrm{M}_{s}, G\right) \rightarrow \mathrm{K}_{*}\left(X_{\leq s}, G\right)$ along the embedding $X_{\leq s}=X_{\leq s} \times\{0\} \hookrightarrow X_{\leq s} \times \mathbb{A}^{1} \hookrightarrow \mathrm{M}_{s}$ is surjective. Consider now the open embedding $X_{\leq t} \subseteq{ }^{X_{\leq s}}$ (for $s \geq t$ ): the pullback $\mathrm{K}_{*}\left(X_{\leq s}, G\right) \rightarrow$ $\mathrm{K}_{*}\left(X_{\leq t}, G\right)$ is also surjective, by $\mathrm{K}$-rigidity (see [Ve-Vi-2, Sect. 4], particularly Definition 4.1, Propositions 4.3 and 4.6). From the commutative diagram

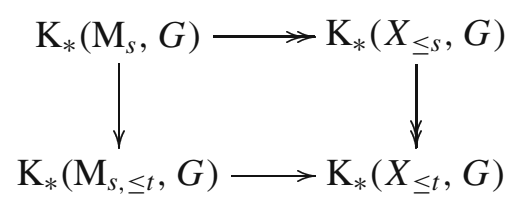


we conclude that the restriction $\mathrm{K}_{*}\left(\mathrm{M}_{s, \leq t}, G\right) \rightarrow \mathrm{K}_{*}\left(X_{\leq t}, G\right)$ is surjective, so we have an exact sequence

$$
0 \rightarrow \mathrm{K}_{*}\left(\mathrm{~N}_{s, \leq t}, G\right) \rightarrow \mathrm{K}_{*}\left(\mathrm{M}_{s, \leq t}, G\right) \rightarrow \mathrm{K}_{*}\left(X_{\leq t}, G\right) \rightarrow 0 .
$$

This allows to define specialization maps

$$
\mathrm{Sp}_{X, s}^{\leq t} \stackrel{\text { def }}{=} \operatorname{Sp}_{\mathrm{N}_{s, \leq t}}^{\mathrm{M}_{s, \leq t}}: \mathrm{K}_{*}\left(X_{\leq t}, G\right) \rightarrow \mathrm{K}_{*}\left(\mathrm{~N}_{s, \leq t}, G\right) .
$$

To define $\mathrm{Sp}_{X, s}^{t}$ consider the commutative diagram with exact rows

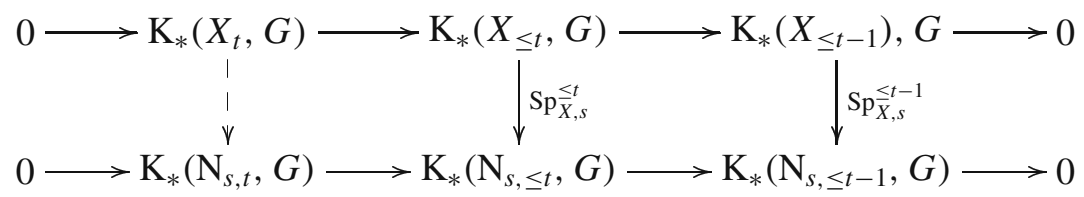

(the commutativity of the second square follows easily from functoriality of pullbacks).

Definition 1. The specialization homomorphism

$$
\mathrm{Sp}_{X, s}^{t}: \mathrm{K}_{*}\left(X_{t}, G\right) \rightarrow \mathrm{K}_{*}\left(\mathrm{~N}_{s, t}, G\right)
$$

is the unique dotted arrow that fits in the diagram above.

These coincide with the usual specialization homomorphisms for $\mathrm{K}_{0}$. This is clear for the $\mathrm{Sp}_{X . S}^{\leq t}$. For $\mathrm{Sp}_{X, S}^{t}$ it follows from the fact the cartesian diagram

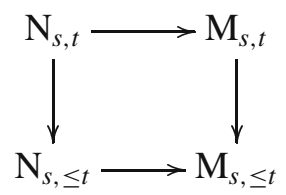

is Tor-independent (because both rows are regular embeddings of codimension 1), and from the following Lemma.

Lemma 1. If

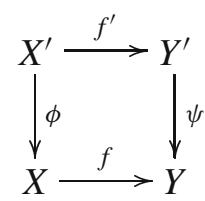


is a Tor-independent cartesian square of regular algebraic spaces with an action of $G$, where $f$ is a closed embedding. Then the diagram

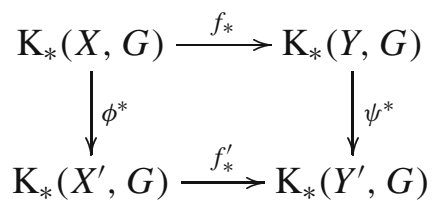

commutes.

The proof that starts at the bottom of p. 11 of [Ve-Vi-2] is general enough.

Now we have to check compatibility of specializations.

Lemma 2. Denote by $i$ the inclusion of $X_{t}$ in $X_{\leq t}$ and by $i^{\prime}$ that of $\mathrm{N}_{s, t}$ in $\mathrm{N}_{s, \leq t}$. Then the diagram

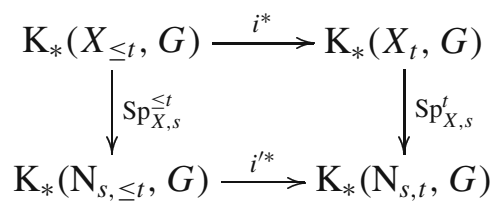

commutes.

Proof. By the definition of $\mathrm{Sp}_{X, s}^{t}$, we need to check that

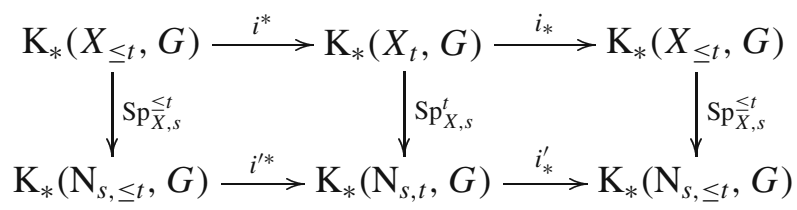

commutes. By the projection formula (see [Ve-Vi, Proposition A.5]) we see that the group homomorphisms $i_{*} i^{*}$ and $i_{*}^{\prime} i^{*}$ are multiplications by

$$
\left[i_{*} \mathcal{O}_{X_{t}}\right] \in \mathrm{K}_{*}\left(X_{\leq t}, G\right) \text { and }\left[i_{*} \mathcal{O}_{\mathrm{N}_{s, t}}\right] \in \mathrm{K}_{*}\left(\mathrm{~N}_{s, \leq t}, G\right)
$$

respectively: so we have to prove that the diagram

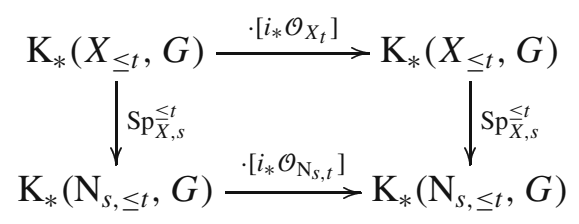

commutes. Since $\mathrm{Sp}_{\bar{X}, s}^{\leq t}$ is a ring homomorphism, this is equivalent to saying that

$$
\operatorname{Sp}_{\bar{X}, s}^{\leq t}\left[i_{*} \mathcal{O}_{X_{t}}\right]=\left[i_{*} \mathcal{O}_{\mathrm{N}_{s, t}}\right] \in \mathrm{K}_{0}\left(\mathrm{~N}_{s, \leq t}, G\right)
$$


But $\left[i_{*} \mathcal{O}_{X_{t}}\right]$ is the restriction of $\left[i_{*} \mathcal{O}_{\mathrm{M}_{s, t}}\right] \in \mathrm{K}_{0}\left(\mathrm{M}_{s, \leq t}, G\right)$, so we have to show that the restriction of $\left[i_{*} \mathcal{O}_{\mathrm{M}_{s, t}}\right]$ to $\mathrm{N}_{s, \leq t}$ is $\left[i_{*} \mathcal{O}_{\mathrm{N}_{s, t}}\right]$; and this follows immediately from the fact that the square

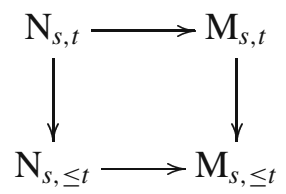

is cartesian and Tor-independent, by Lemma 1.

With this definition, and the compatibility property proved above, everything goes through in Sects. 4, 5 and 6. For the theory of Sect. 7 to work, we need to define specialization maps

$$
\mathrm{K}_{*}\left(X^{(\tau)}, G\right) \rightarrow \mathrm{K}_{*}\left(\mathrm{~N}_{s}^{(\tau)}, G\right)
$$

when $\tau$ is a diagonalizable subgroup scheme of $G$ and $s$ is an integer with $s \geq \operatorname{dim} \tau$ (see the bottom of p. 39).The cartesian diagram of embeddings

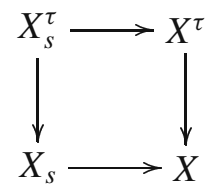

yields an embedding of $G$-spaces $\mathrm{N}_{X_{s}^{\tau}} X^{\tau} \hookrightarrow \mathrm{N}_{s}$; since $\tau$ acts trivially on $\mathrm{N}_{X_{s}^{\tau}} X^{\tau}$ we get an embedding $\mathrm{N}_{X_{s}^{\tau}} X^{\tau} \hookrightarrow \mathrm{N}_{s}^{\tau}$.

Lemma 3. The embedding $\mathrm{N}_{X_{s}^{\tau}} X^{\tau} \hookrightarrow \mathrm{N}_{s}^{\tau}$ is an isomorphism.

Proof. Consider the natural closed embedding of deformations to the normal bundle

$$
\mathrm{M}_{X_{s}^{\tau}}^{0} X^{\tau} \hookrightarrow\left(\mathrm{M}_{s}\right)^{\tau}
$$

generically, that is, over $\mathbb{A}^{1}$, they coincide. On the other hand it follows from Proposition 3.6 in the paper that the inverse image of $\mathbb{A}^{1}$ in $\left(\mathrm{M}_{s}\right)^{\tau}$ is scheme-theoretically dense in $\left(\mathrm{M}_{s}\right)^{\tau}$, and this shows that this embedding is an isomorphism. Since the fibers over $\infty$ of $\mathbf{M}_{X_{s}^{\tau}}^{0} X^{\tau}$ and $\left(\mathbf{M}_{s}\right)^{\tau}$ are $\mathrm{N}_{X_{s}^{\tau}} X^{\tau}$ and $\mathrm{N}_{s}^{\tau}$ respectively, this concludes the proof.

Now set $t=\operatorname{dim} \tau$, so that $X^{(\tau)} \stackrel{\text { def }}{=} X_{t}^{\tau}$. Then we get a specialization map

$$
\begin{aligned}
\mathrm{Sp}_{X, s}^{\tau} \stackrel{\text { def }}{=} \mathrm{Sp}_{X^{\tau}, s}^{t}: \mathrm{K}_{*}\left(X_{t}^{\tau}, G\right) & =\mathrm{K}_{*}\left(X^{(\tau)}, G\right) \rightarrow \mathrm{K}_{*}\left(\left(\mathrm{~N}_{s}^{\tau}\right)_{t}, G\right) \\
& =\mathrm{K}_{*}\left(\mathrm{~N}_{s}^{(\tau)}, G\right)
\end{aligned}
$$


that is exactly what we want. This allows to define the specialization map

$$
\mathrm{Sp}_{X, \sigma}^{\tau}: \mathrm{K}_{*}\left(X_{t}^{\tau}, G\right) \rightarrow \mathrm{K}_{*}\left(\mathrm{~N}_{\sigma}^{(\tau)}, G\right)
$$

for any pair of dual cyclic subgroups $\sigma$ and $\tau$, with $\tau \prec \sigma$, as on p. 41 of the paper, by composing $\mathrm{Sp}_{X^{\tau}, S}^{t}$ with the restriction homomorphism $\mathrm{K}_{*}\left(\mathrm{~N}_{s}^{(\tau)}, G\right) \rightarrow \mathrm{K}_{*}\left(\mathrm{~N}_{\sigma}^{(\tau)}, G\right)$. Note that $X^{(\tau)}=X_{\leq t}^{\tau}, \mathrm{N}_{s}^{(\tau)}=\left(\mathrm{N}_{s}^{\tau}\right)_{\leq t}$, and $\mathrm{Sp}_{X, s}^{\tau}$ can also be identified with $\mathrm{Sp}_{X^{\tau}, s}^{\leq t}$; therefore $\mathrm{Sp}_{X, s}^{\tau}$ and $\mathrm{Sp}_{X, \sigma}^{\tau}$ are ring homomorphisms.

Further corrections. Here we correct a few typos that we have noticed since the publication of the article.

In the statement of Proposition 1.1, "scheme" should be replaced by "algebraic space".

The are several typos in the diagrams on p. 42 :

(1) $\prod \mathrm{Sp}_{X, s}^{s-1}$ should be replaced by $\mathrm{Sp}_{X, s}^{s-1}$,

(2) $\mathrm{Sp}_{X, s}^{\tau}$ by $\prod \mathrm{Sp}_{X, s}^{\tau}$,

(3) $\mathrm{N}^{(\tau)}$ by $\mathrm{N}_{s}^{(\tau)}$ and

(4) $\mathrm{K}_{*}\left(\mathrm{~N}_{\sigma}^{(\tau)}, G\right)$ by $\mathrm{K}_{*}\left(\mathrm{~N}_{\sigma}^{(\tau)}, G\right)_{\tau}$

Finally, in the statement of Lemma 4.9, "linearly independent elements" should read "pairwise linearly independent elements" (we owe this also to Amnon Neeman).

Acknowledgements. We are very much in debt with Amnon Neeman who read our paper carefully and kindly pointed out the problem to us.

\section{References}

[SGA6] Berthelot, P., Grothendieck, A., Illusie, L.: Théorie des intersections et théorème de Riemann-Roch. Lect. Notes Math., vol. 225. Berlin: Springer 1971

[Ful] Fulton, W.: Intersection theory. Second edition. Berlin: Springer 1998

[Ve-Vi] Vezzosi, G., Vistoli, A.: Higher algebraic K-theory of group actions with finite stabilizers. Duke. Math. J. 113, 1-55 (2002)

[Ve-Vi-2] Vezzosi, G., Vistoli, A.: Higher algebraic K-theory for actions of diagonalizable groups. Invent. Math. 153, 1-44 (2003) 